\title{
Semirings and Formal Power Series
}

\author{
Manfred Droste ${ }^{1}$ and Werner Kuich ${ }^{2}$ \\ 1 Institut für Informatik, Universität Leipzig, D-04009 Leipzig, Germany \\ droste@informatik.uni-leipzig.de \\ 2 Institut für Diskrete Mathematik und Geometrie, Technische Universität Wien, \\ A-1040 Wien, Austria \\ www.dmg.tuwien.ac.at/kuich
}

\section{Introduction}

It is the goal of this chapter to present basic foundations for the theory of weighted automata: semirings and formal power series.

Weighted automata are classical automata in which the transitions carry weights. These weights may model, e.g., the cost involved when executing the transition, the amount of resources or time needed for this, or the probability or reliability of its successful execution. In order to obtain a uniform model of weighted automata for different realizations of weights and their computations, the weight structures are often modeled as semirings. A semiring consists of a set with two operations addition and multiplication satisfying certain natural axioms like associativity, commutativity and distributivity, just like the natural numbers with their laws for sums and products. The behaviour of weighted automata can then be defined as a function associating to each word the total weight of its execution, see later chapters $[13,39]$ of this handbook.

Any function from the free monoid $\Sigma^{*}$ of all words over a given alphabet $\Sigma$ into a semiring $S$ is called a formal power series. It is important to notice that each language over $\Sigma$ can be viewed as a formal power series over $\mathbb{B}$ and $\Sigma^{*}$ (by identifying the language with its characteristic series). Therefore formal power series form a generalization of formal languages, and, similarly, weighted automata generalize classical automata. For other semirings (like the natural or real numbers), formal power series can be viewed as weighted, multivalued or quantified languages in which each word is assigned a weight, a number, or some quantity.

In this chapter, we will present the basics of the theory of semirings and formal power series as far as they are used in the forthcoming chapters of this Handbook. Now we give a summary of the contents of this chapter.

First, we consider various particular monoids and semirings. Many semirings (like the natural numbers) carry a natural order. Also, when generalizing the star operation (= Kleene iteration) from languages to formal power series, 
important questions on the existence of infinite sums arise. This leads to the notions of ordered, complete or continuous monoids and semirings. Besides these, we will consider the related concepts of star semirings and Conway semirings, and also locally finite semirings.

Next, we introduce formal power series, especially locally finite families of power series and cycle-free power series. It is a basic result that the collection of all formal power series over a given semiring and an alphabet can be endowed with addition and Cauchy multiplication yielding again the structure of a semiring, as well as with several further useful operations like the Hadamard product or the Hurwitz (shuffle) product. We prove that, under suitable assumptions, certain equalities involving the Kleene-star of elements are valid. Moreover, various important properties of the underlying semiring transfer to the semiring of formal power series. In particular, this includes properties like being ordered, complete, continuous or Conway. We also consider morphisms between semirings of formal power series.

As is well-known, the set of transitions of a classical finite automaton can be uniformly represented by matrices with entries 0 or 1 . A similar representation is also easily possible for the transitions of a weighted automaton: here the matrices have entries from the underlying semiring, viz. the weights of the transitions. This yields very compact representations of weighted automata and often very concise algebraic proofs about their behaviors. We prove a theorem on (infinite) matrices central for automata theory: In a complete star semiring, the blocks of the star of a matrix can be represented by applying rational operations to the blocks of the matrix. Moreover, the Kronecker (tensor) product of matrices is considered.

Finally, we consider cycle-free equations. They have a unique solution and can be used to show that two expressions represent the same formal power series. Again we obtain results on how to compute the blocks of the star of a matrix, but now for arbitrary semirings, by imposing restrictions on the matrix.

In the literature, a number of authors have dealt with the interplay between semirings, formal power series and automata theory. The following books and surveys deal with this topic: Berstel [2], Berstel, Reutenauer [3], Bloom, Ésik [4], Carré [5], Conway [6], Eilenberg [9], Ésik, Kuich [10], Kuich [29], Kuich, Salomaa [30], Sakarovitch [38], Salomaa, Soittola [40], Wechler [41].

Further books on semirings and formal power series are Golan [16] and Hebisch, Weinert [21]. Głazek [14] is a bibliography on semirings and formal power series.

Some ideas and formulations of this presentation originate from Kuich, Salomaa [30] and Ésik, Kuich [12]. 


\section{Monoids and semirings}

In this section we consider monoids and semirings. The definitions and results on monoids and semirings are mainly due to Bloom, Ésik [4], Eilenberg [9], Goldstern [17], Karner [23, 24], Krob [26, 27], Kuich [28, 29], Kuich, Salomaa [30], Manes, Arbib [32], Sakarovitch [37]. Our notion of continuous monoids and semirings is a specialization of the continuous algebras as defined, e.g., in Guessarian [18], Goguen, Thatcher, Wagner, Wright [15], Adámek, Nelson, Reiterman [1].

A monoid consists of a non-empty set $M$, an associative binary operation . on $M$ and a neutral element 1 such that $m \cdot 1=1 \cdot m=m$ for every $m \in M$. A monoid $M$ is called commutative if $m_{1} \cdot m_{2}=m_{2} \cdot m_{1}$ for every $m_{1}, m_{2} \in M$. The binary operation is usually denoted by juxtaposition and often called product.

If the operation and the neutral element of $M$ are understood, then we denote the monoid simply by $M$. Otherwise, we use the triple notation $\langle M, \cdot, 1\rangle$. A commutative monoid $M$ is often denoted by $\langle M,+, 0\rangle$.

The most important type of a monoid in our considerations is the free monoid $\Sigma^{*}$ generated by a non-empty set $\Sigma$. It has all the (finite) words over $\Sigma$

$$
x_{1} \ldots x_{n}, \text { with } x_{i} \in \Sigma, 1 \leq i \leq n, n \geq 0,
$$

as its elements, and the product $w_{1} \cdot w_{2}$ is formed by writing the string $w_{2}$ immediately after the string $w_{1}$. The neutral element of $\Sigma^{*}$ (the case $n=0$ ), also referred to as the empty word, is denoted by $\varepsilon$.

The elements of $\Sigma$ are called letters or symbols. The set $\Sigma$ itself is called an alphabet. The length of a word $w=x_{1} \ldots x_{n}, n \geq 0$, in symbols $|w|$, is defined to be $n$.

A morphism $h$ of a monoid $M$ into a monoid $M^{\prime}$ is a mapping $h: M \rightarrow M^{\prime}$ compatible with the neutral elements and operations in $\langle M, \cdot 1\rangle$ and $\left\langle M^{\prime}, \circ, 1^{\prime}\right\rangle$, i. e., $h(1)=1^{\prime}$ and $h\left(m_{1} \cdot m_{2}\right)=h\left(m_{1}\right) \circ h\left(m_{2}\right)$ for all $m_{1}, m_{2} \in M$.

If $\Sigma$ is an alphabet and $\langle M, \cdot, 1\rangle$ is any monoid, then every mapping $h$ : $\Sigma \rightarrow M$ can be uniquely extended to a morphism $h^{\sharp}: \Sigma^{*} \rightarrow M$ by putting $h^{\sharp}(\varepsilon)=1$ and $h^{\sharp}\left(x_{1} x_{2} \ldots x_{n}\right)=h\left(x_{1}\right) \cdot h\left(x_{2}\right) \cdot \ldots \cdot h\left(x_{n}\right)$ for any $x_{1}, \ldots, x_{n} \in \Sigma$, $n \geq 1$. Usually $h^{\sharp}$ is again denoted by $h$.

Next we consider monoids with particular properties, like carrying an order or having an infinite sum operation. For our purposes, it suffices to consider commutative monoids. A commutative monoid $\langle M,+, 0\rangle$ is called idempotent, if $m+m=m$ for all $m \in M$, and it is called ordered if it is equipped with a partial order $\leq$ preserved by the + operation. An ordered monoid $M$ is positively ordered, if $m \geq 0$ for each $m \in M$. A commutative monoid $\langle M,+, 0\rangle$ is called naturally ordered if the relation $\sqsubseteq$ defined by: $m_{1} \sqsubseteq m_{2}$ if there exists an $m$ such that $m_{1}+m=m_{2}$, is a partial order. Clearly, this is the case, i.e., $\sqsubseteq$ is antisymmetric, iff whenever $m, m^{\prime}, m^{\prime \prime} \in M$ with $m+m^{\prime}+m^{\prime \prime}=m$, then $m+m^{\prime}=m$. Then in particular $M$ is positively 
ordered. We note that if $\langle M,+, 0\rangle$ is idempotent, then $M$ is naturally ordered and for any $m_{1}, m_{2} \in M$ we have $m_{1}+m_{2}=\sup \left\{m_{1}, m_{2}\right\}$ in $\langle M$, $\sqsubseteq\rangle$, further, $m_{1} \sqsubseteq m_{2}$ iff $m_{1}+m_{2}=m_{2}$. Morphisms of ordered monoids are monoid morphisms which preserve the order.

If $I$ is an index set, an infinitary sum operation $\sum_{I}: M^{I} \rightarrow M$ associates with every family $\left(m_{i} \mid i \in I\right)$ of elements of $M$ an element $\sum_{i \in I} m_{i}$ of $M$. A monoid $\langle M,+, 0\rangle$ is called complete if it has infinitary sum operations $\sum_{I}$ (for any index set $I$ ) such that the following conditions are satisfied:

(i) $\sum_{i \in \emptyset} m_{i}=0, \sum_{i \in\{j\}} m_{i}=m_{j}, \sum_{i \in\{j, k\}} m_{i}=m_{j}+m_{k}$, for $j \neq k$,

(ii) $\sum_{j \in J}\left(\sum_{i \in I_{j}} m_{i}\right)=\sum_{i \in I} m_{i}$, if $\bigcup_{j \in J} I_{j}=I$ and $I_{j} \cap I_{j^{\prime}}=\emptyset$ for $j \neq j^{\prime}$.

A morphism of complete monoids is a monoid morphism preserving all sums. Note that any complete monoid is commutative.

Recall that a non-empty subset $D$ of a partially ordered set $P$ is called directed if each pair of elements of $D$ has an upper bound in $D$.

A positively ordered commutative monoid $\langle M,+, 0\rangle$ is called a continuous monoid if each directed subset of $M$ has a least upper bound and the + operation preserves the least upper bound of directed sets, i.e., when

$$
m+\sup D=\sup (m+D),
$$

for all directed sets $D \subseteq M$ and for all $m \in M$. Here, $m+D$ is the set $\{m+d \mid d \in D\}$.

It is known that a positively ordered commutative monoid $M$ is continuous iff each chain in $M$ has a least upper bound and the + operation preserves least upper bounds of chains, i. e., when $m+\sup C=\sup (m+C)$ holds for all non-empty chains $C$ in $M$. (See Markowsky [33].)

Proposition 2.1. Any continuous monoid $\langle M,+, 0\rangle$ is a complete monoid equipped with the following sum operation:

$$
\sum_{i \in I} m_{i}=\sup \left\{\sum_{i \in F} m_{i} \mid F \subseteq I, F \text { finite }\right\},
$$

for all index sets $I$ and all families $\left(m_{i} \mid i \in I\right)$ in $M$.

A function $f: P \rightarrow Q$ between partially ordered sets is continuous if it preserves the least upper bound of any directed set, i.e., when $f(\sup D)=$ $\sup f(D)$, for all directed sets $D \subseteq P$ such that $\sup D$ exists. It follows that any continuous function preserves the order. A morphism of continuous monoids is defined to be a monoid morphism which is a continuous function. Clearly, any morphism between continuous monoids is a complete monoid morphism.

A semiring is a set $S$ together with two binary operations + and $\cdot$ and two constant elements 0 and 1 such that

(i) $\langle S,+, 0\rangle$ is a commutative monoid,

(ii) $\langle S, \cdot, 1\rangle$ is a monoid, 
(iii) the distributivity laws $s_{1} \cdot\left(s_{2}+s_{3}\right)=s_{1} \cdot s_{2}+s_{1} \cdot s_{3}$ and $\left(s_{1}+s_{2}\right) \cdot s_{3}=$ $s_{1} \cdot s_{3}+s_{2} \cdot s_{3}$ hold for every $s_{1}, s_{2}, s_{3} \in S$,

(iv) $0 \cdot s=s \cdot 0=0$ for every $s \in S$.

A semiring $S$ is called commutative if $s_{1} \cdot s_{2}=s_{2} \cdot s_{1}$ for every $s_{1}, s_{2} \in S$. Further, $S$ is called idempotent if $\langle S,+, 0\rangle$ is an idempotent monoid. By the distributivity law, this holds iff $1+1=1$.

If the operations and the constant elements of $S$ are understood, then we denote the semiring simply by $S$. Otherwise, we use the notation $\langle S,+, \cdot, 0,1\rangle$. In the sequel, $S$ will denote a semiring.

Intuitively, a semiring is a ring (with unity) without subtraction. A typical example is the semiring of nonnegative integers $\mathbb{N}$. A very important semiring in connection with language theory is the Boolean semiring $\mathbb{B}=\{0,1\}$ where $1+1=1 \cdot 1=1$. Clearly, all rings (with unity), as well as all fields, are semirings, e. g., the integers $\mathbb{Z}$, rationals $\mathbb{Q}$, reals $\mathbb{R}$, complex numbers $\mathbb{C}$ etc.

Let $\mathbb{N}^{\infty}=\mathbb{N} \cup\{\infty\}$ and $\overline{\mathbb{N}}=\mathbb{N} \cup\{-\infty, \infty\}$. Then $\left\langle\mathbb{N}^{\infty},+, \cdot, 0,1\right\rangle$, $\left\langle\mathbb{N}^{\infty}, \min ,+, \infty, 0\right\rangle$ and $\langle\overline{\mathbb{N}}, \max ,+,-\infty, 0\rangle$, where + , $\cdot$ min and max are defined in the obvious fashion (observe that $0 \cdot \infty=\infty \cdot 0=0$ and $(-\infty)+\infty=-\infty)$, are semirings.

Let $\mathbb{R}_{+}=\{a \in \mathbb{R} \mid a \geq 0\}, \mathbb{R}_{+}^{\infty}=\mathbb{R}_{+} \cup\{\infty\}$ and $\overline{\mathbb{R}}_{+}=\mathbb{R}_{+} \cup\{-\infty, \infty\}$. Then $\left\langle\mathbb{R}_{+},+, \cdot, 0,1\right\rangle,\left\langle\mathbb{R}_{+}^{\infty},+, \cdot, 0,1\right\rangle$ and $\left\langle\mathbb{R}_{+}^{\infty}, \min ,+, \infty, 0\right\rangle$ are semirings. The semirings $\left\langle\mathbb{N}_{+}^{\infty}, \min ,+, \infty, 0\right\rangle$ and $\left\langle\mathbb{R}_{+}^{\infty}, \min ,+, \infty, 0\right\rangle$ are called tropical semirings or min-plus-semirings. Similarly, the semirings $\langle\overline{\mathbb{N}}, \max ,+,-\infty, 0\rangle$ and $\left\langle\mathbb{\mathbb { R }}_{+}, \max ,+,-\infty, 0\right\rangle$ are called max-plus semirings or arctic semirings. A further example is provided by the semiring $\langle[0,1], \max , \cdot, 0,1\rangle$, called the Viterbi semiring in probabilistic parsing.

We note that the tropical and the arctic semirings are very often employed in optimization problems of networks, cf., e.g., Heidergott, Olsder, van der Woude [22].

Let $\Sigma$ be a finite alphabet. Then each subset of $\Sigma^{*}$ is called a formal language over $\Sigma$. We define, for formal languages $L_{1}, L_{2} \subseteq \Sigma^{*}$, the product of $L_{1}$ and $L_{2}$ by

$$
L_{1} \cdot L_{2}=\left\{w_{1} w_{2} \mid w_{1} \in L_{1}, w_{2} \in L_{2}\right\} .
$$

Then $\left\langle 2^{\Sigma^{*}}, \cup, \cdot, \emptyset,\{\varepsilon\}\right\rangle$ is a semiring, called the semiring of formal languages over $\Sigma$. Here $2^{U}$ denotes the power set of a set $U$ and $\emptyset$ denotes the empty set.

If $U$ is a set, $2^{U \times U}$ is the set of binary relations over $U$. Define, for two relations $R_{1}$ and $R_{2}$, the product $R_{1} \cdot R_{2} \subseteq U \times U$ by

$$
\begin{aligned}
& R_{1} \cdot R_{2}=\left\{\left(u_{1}, u_{2}\right) \mid \text { there exists } u \in U\right. \text { such that } \\
& \left.\qquad\left(u_{1}, u\right) \in R_{1} \text { and }\left(u, u_{2}\right) \in R_{2}\right\}
\end{aligned}
$$

and, furthermore, define

$$
\Delta=\{(u, u) \mid u \in U\}
$$


Then $\left\langle 2^{U \times U}, \cup, \cdot,, \Delta\right\rangle$ is a semiring, called the semiring of binary relations over $U$.

Further semirings are the chain of nonnegative reals $\left\langle\mathbb{R}_{+}^{\infty}, \max , \min , 0, \infty\right\rangle$ and any Boolean algebra, in particular the power set Boolean algebras $\left\langle 2^{U}, \cup, \cap, \emptyset, U\right\rangle$ where $U$ is any set. These examples can be generalized as follows. Recall that a partially ordered set $\langle L, \leq\rangle$ is a lattice if for any two elements $a, b \in L$, the least upper bound $a \vee b=\sup \{a, b\}$ and the greatest lower bound $a \wedge b=\inf \{a, b\}$ exist in $\langle L, \leq\rangle$. A lattice $\langle L, \leq\rangle$ is distributive, if $a \wedge(b \vee c)=(a \wedge b) \vee(a \wedge c)$ for all $a, b, c \in L$; and bounded, if $L$ contains a smallest element, denoted 0 , and a greatest element, denoted 1. Now let $\langle L, \leq\rangle$ be any bounded distributive lattice. Then $\langle L, \vee, \wedge, 0,1\rangle$ is a semiring. Since any distributive lattice $L$ also satisfies the dual law $a \vee(b \wedge c)=(a \vee b) \wedge(a \vee c)$ for all $a, b, c \in L$, the structure $\langle L, \wedge, \vee, 1,0\rangle$ is also a semiring. Such semirings are often used for fuzzy automata, see Chapter 12 [36] of this book. Another semiring is the Eukasiewicz semiring $\langle[0,1], \max , \otimes, 0,1\rangle$ where $a \otimes b=\max \{0, a+b-1\}$ which occurs in multivalued logic (see Hájek [19]).

Recall that in formal language theory, the Kleene-iteration $L^{*}$ of a language $L \subseteq \Sigma^{*}$ is defined by $L^{*}=\bigcup_{n>0} L^{n}$. Later on, we wish to extend this star operation to formal power series (i.e. functions) $r: \Sigma^{*} \rightarrow S$ where $S$ is a semiring. For this, it will be useful to know which semirings carry such a star operation like the semiring of formal languages. We will call a star semiring any semiring equipped with an additional unary operation *. The following semirings are star semirings:

(i) The Boolean semiring $\left\langle\mathbb{B},+, \cdot,{ }^{*}, 0,1\right\rangle$ with $0^{*}=1^{*}=1$.

(ii) The semiring $\left\langle\mathbb{N}^{\infty},+, \cdot,{ }^{*}, 0,1\right\rangle$ with $0^{*}=1$ and $a^{*}=\infty$ for $a \neq 0$.

(iii) The semiring $\left\langle\mathbb{R}_{+}^{\infty},+, \cdot,^{*}, 0,1\right\rangle$ with $a^{*}=1 /(1-a)$ for $0 \leq a<1$ and $a^{*}=\infty$ for $a \geq 1$.

(iv) The tropical semirings $\left\langle\mathbb{R}_{+}^{\infty}, \min ,+,{ }^{*}, \infty, 0\right\rangle$ and $\left\langle\mathbb{N}^{\infty}, \min ,+{ }^{*}, \infty, 0\right\rangle$ with $a^{*}=0$ for all $a \in \mathbb{R}_{+}^{\infty}$ resp. all $a \in \mathbb{N}^{\infty}$.

(v) The arctic semirings $\left\langle\overline{\mathbb{R}}_{+}, \max ,+,{ }^{*},-\infty, 0\right\rangle$ and $\left\langle\overline{\mathbb{N}}, \max ,+,{ }^{*},-\infty, 0\right\rangle$ with $(-\infty)^{*}=0^{*}=0$ and $a^{*}=\infty$ for $a>0$.

(vi) The semiring $\left\langle 2^{\Sigma^{*}}, \cup, \cdot,{ }^{*}, \emptyset,\{\varepsilon\}\right\rangle$ of formal languages over a finite alphabet $\Sigma$, as noted before, with $L^{*}=\bigcup_{n \geq 0} L^{n}$ for all $L \subseteq \Sigma^{*}$.

(vii) The semiring $\left\langle 2^{U \times U}, \cup, \cdot,^{*}, \emptyset, \Delta\right\rangle$ of binary relations over $U$ with $R^{*}=$ $\bigcup_{n>0} R^{n}$ for all $R \subseteq U \times U$. The relation $R^{*}$ is called the reflexive and transitive closure of $R$, i. e., the smallest reflexive and transitive binary relation over $S$ containing $R$.

(viii) The Eukasiewiez semiring $\left\langle[0,1], \max , \otimes,{ }^{*}, 0,1\right\rangle$ with $a^{*}=1$ for all $a \in$ $[0,1]$.

(ix) The idempotent naturally ordered commutative semiring $\left\langle\{0,1, a, \infty\},+, \cdot,^{*}, 0,1\right\rangle$, with $0 \sqsubseteq 1 \sqsubseteq a \sqsubseteq \infty, a \cdot a=a, 0^{*}=1^{*}=1$, $a^{*}=\infty^{*}=\infty$. 
(x) The bounded distributive lattice semiring $\left\langle L, \vee, \wedge,{ }^{*}, 0,1\right\rangle$ with $a^{*}=1$ for all $a \in L$.

The semirings (i) $-(\mathrm{v})$ and (viii) $-(\mathrm{x})$ are commutative, the semirings (i), (iv)-(x) are idempotent.

A semiring $\langle S,+, \cdot, 0,1\rangle$ is called ordered if $\langle S,+, 0\rangle$ is an ordered monoid and multiplication with elements $s \geq 0$ preserves the order; it is positively ordered, if, furthermore, $\langle S,+, 0\rangle$ is positively ordered. When the order on $S$ is the natural order, $\langle S,+, \cdot, 0,1\rangle$ is automatically a positively ordered semiring.

A semiring $\langle S,+, \cdot, 0,1\rangle$ is called complete if $\langle S,+, 0\rangle$ is a complete monoid and the following distributivity laws are satisfied (see Bloom, Ésik [4], Conway [6], Eilenberg [9], Kuich [29]):

$$
\sum_{i \in I}\left(s \cdot s_{i}\right)=s \cdot\left(\sum_{i \in I} s_{i}\right), \quad \sum_{i \in I}\left(s_{i} \cdot s\right)=\left(\sum_{i \in I} s_{i}\right) \cdot s .
$$

This means that a semiring $S$ is complete if it is possible to define "infinite sums" (i) that are an extension of the finite sums, (ii) that are associative and commutative and (iii) that satisfy the distributivity laws.

In complete semirings for each element $s$ we can define the star $s^{*}$ of $s$ by

$$
s^{*}=\sum_{j \geq 0} s^{j},
$$

where $s^{0}=1$ and $s^{j+1}=s \cdot s^{j}=s^{j} \cdot s$ for $j \geq 0$. Hence, with this star operation, each complete semiring is a star semiring called a complete star semiring. The semirings (i)-(viii) are complete star semirings. The semiring (ix) is complete, but it violates the above equation for $s=a$, hence it is not a complete star semiring. The distributive lattice semiring $L$ satisfies $a^{*}=\bigvee_{j \geq 0} a^{j}$ for each $a \in L$, but is not necessarily complete. It is a complete semiring iff $(L, \vee, \wedge)$ is a join-continuous complete lattice, i.e., any subset of $L$ has a supremum in $L$ and $a \wedge \bigvee_{i \in I} a_{i}=\bigvee_{i \in I}\left(a \wedge a_{i}\right)$ for any subset $\left\{a_{i} \mid i \in I\right\}$ of $L$.

A semiring $\langle S,+, \cdot, 0,1\rangle$ is called continuous if $\langle S,+, 0\rangle$ is a continuous monoid and if multiplication is continuous, i.e.,

$$
s \cdot\left(\sup _{i \in I} s_{i}\right)=\sup _{i \in I}\left(s \cdot s_{i}\right) \quad \text { and } \quad\left(\sup _{i \in I} s_{i}\right) \cdot s=\sup _{i \in I}\left(s_{i} \cdot s\right)
$$

for all directed sets $\left\{s_{i} \mid i \in I\right\}$ and $s \in S$ (see Bloom, Ésik [4]). It follows that the distributivity laws hold for infinite sums:

$$
s \cdot\left(\sum_{i \in I} s_{i}\right)=\sum_{i \in I}\left(s \cdot s_{i}\right) \quad \text { and } \quad\left(\sum_{i \in I} s_{i}\right) \cdot s=\sum_{i \in I}\left(s_{i} \cdot s\right)
$$

for all families $\left(s_{i} \mid i \in I\right)$.

Proposition 2.2. Any continuous semiring is complete. 
All the semirings in (i)-(ix) are continuous.

We now consider two equations that are important in automata theory. Let $S$ be a star semiring. Then, for $a, b \in S$,

(i) the sum star identity is valid for $a$ and $b$ if $(a+b)^{*}=\left(a^{*} b\right)^{*} a^{*}$;

(ii) the product star identity is valid for $a$ and $b$ if $(a b)^{*}=1+a(b a)^{*} b$.

If the sum star identity (resp. the product star identity) is valid for all $a, b \in S$ then we say that the sum star identity (resp. the product star identity) is valid (in the star semiring $S$ ).

A Conway semiring is now a star semiring in which the sum star identity and the product star identity are valid (see Conway [6], Bloom, Ésik [4]). All the star semirings in (i) $-(\mathrm{x})$ are Conway semirings. The semiring $\left\langle\mathbb{Q}_{+}^{\infty},+, \cdot{ }^{*}, 0,1\right\rangle$, with $\mathbb{Q}_{+}^{\infty}=\mathbb{R}_{+}^{\infty} \cap(\mathbb{Q} \cup\{\infty\})$ and operations defined as in (iii), is a Conway semiring (since the sum star identity and product star identity hold in $\mathbb{R}_{+}^{\infty}$ ) but is not complete. Now we have:

Proposition 2.3. Let $S$ be a star semiring. Then $S$ is a Conway semiring iff, for all $a, b \in S$,

(i) $(a+b)^{*}=\left(a^{*} b\right)^{*} a^{*}$,

(ii) $(a b)^{*} a=a(b a)^{*}$,

(iii) $a^{*}=1+a a^{*}=1+a^{*} a$.

Proof. If $S$ is a Conway semiring, we obtain (iii) from the product star identity with $b=1$ resp. $a=1$. Then (ii) follows from the product star identity, distributivity and (iii). Conversely, for the product star identity compute $(a b)^{*}$ by using (iii) and then (ii).

Next we introduce conditions which often simplify the definition or the calculation of the star of elements. A semiring $S$ is $k$-closed, where $k \geq 0$, if for each $a \in S$,

$$
1+a+\cdots+a^{k}=1+a+\cdots+a^{k}+a^{k+1} .
$$

It is called locally closed, if for each $a \in S$ there is an integer $k \geq 0$ such that the above equality is valid. (See Carré [5], Mohri [34], Ésik, Kuich [11], Zhao [42], Zimmermann [43].) If $\langle S,+, \cdot, 0,1\rangle$ is a $k$-closed semiring, then define the star of $a \in S$ by

$$
a^{*}=1+a+\cdots+a^{k} .
$$

An analogous equality defines the star in a locally closed semiring. With this star operation, each $k$-closed (resp. locally closed) semiring is a star semiring called a $k$-closed (resp. locally closed) star semiring. The semirings (i), (iv), (viii), (x) are 0-closed star semirings; the semiring (ix) is a 1-closed semiring, but not a 1-closed star semiring.

Theorem 2.4. Any locally closed star semiring is a Conway semiring. 
We now consider morphisms between semirings. Let $S$ and $S^{\prime}$ be semirings. Then a mapping $h: S \rightarrow S^{\prime}$ is a morphism from $S$ into $S^{\prime}$ if $h(0)=0, h(1)=1$, $h\left(s_{1}+s_{2}\right)=h\left(s_{1}\right)+h\left(s_{2}\right)$ and $h\left(s_{1} \cdot s_{2}\right)=h\left(s_{1}\right) \cdot h\left(s_{2}\right)$ for all $s_{1}, s_{2} \in S$. That is, a morphism of semirings is a mapping that preserves the semiring operations and constants. A bijective morphism is called an isomorphism. For instance, the semirings $\left\langle\mathbb{R}_{+}^{\infty}, \min ,+, \infty, 0\right\rangle$ and $\langle[0,1], \max , \cdot, 0,1\rangle$ are isomorphic via the mapping $x \mapsto e^{-x}$, and the semiring $\left\langle\mathbb{R}_{+}^{\infty}\right.$, max, min, $\left.0, \infty\right\rangle$ is isomorphic to $\langle[0,1], \max , \min , 0,1\rangle$ via the mapping $x \mapsto 1-e^{-x}$. A morphism $h$ of star semirings is a semiring morphism that preserves additionally the star operation, i. e., $h\left(s^{*}\right)=h(s)^{*}$ for all $s \in S$. Similarly, a morphism of ordered (resp. complete, continuous) semirings is a semiring morphism that preserves the order (resp. all sums, all suprema of directed subsets). Note that every continuous semiring is an ordered semiring and every continuous semiring morphism is an ordered semiring morphism.

Complete and continuous semirings are typically infinite. For results on weighted automata, sometimes it is assumed that the underlying semiring is finite or "close" to being finite. A large class of such semirings can be obtained by the notion of local finiteness (which stems from group theory where it is well-known).

A semiring $S$ is locally finite (see Wechler [41], Droste, Gastin [7]) if each finitely generated subsemiring is finite. We note that a semiring $\langle S,+, \cdot, 0,1\rangle$ is locally finite iff both monoids $\langle S,+, 0\rangle$ and $\langle S, \cdot, 1\rangle$ are locally finite. Indeed, if $\langle S,+, 0\rangle$ and $\langle S, \cdot, 1\rangle$ are locally finite and $U$ is a finite subset of $S$, then the submonoid $V$ of $\langle S, \cdot, 1\rangle$ generated by $U$ is finite and the submonoid $W$ of $\langle S,+, 0\rangle$ generated by $V$ is also finite. Now, it is easy to check that $W \cdot W \subseteq W$ and we deduce that the subsemiring of $\langle S,+, \cdot, 0,1\rangle$ generated by $U$ is the finite set $W$.

For instance, if both sum and product are commutative and idempotent, then the semiring is locally finite. Consequently, any bounded distributive lattice $\langle L, \vee, \wedge, 0,1\rangle$ is a locally finite semiring. In particular, the chain $\langle[0,1]$, max, min, 0,1$\rangle$ and any Boolean algebra are locally finite. Further, the Eukasiewiez semiring $\langle[0,1], \max , \otimes, 0,1\rangle$ is locally finite, since its additive and multiplicative monoid are commutative and locally finite. Moreover, each positively ordered locally finite semiring is locally closed, and each positively ordered finite semiring is $k$-closed where $k$ is less than the number of elements of the semiring.

Examples of infinite but locally finite fields are provided by the algebraic closures of the finite fields $\mathbb{Z} / p \mathbb{Z}$ for any prime $p$.

\section{Formal power series}

We now define formal power series (for expositions, see Salomaa, Soittola [40], Kuich, Salomaa [30], Berstel, Reutenauer [3], Sakarovitch [38]). Let $\Sigma$ be an alphabet and $S$ a semiring. Mappings $r$ from $\Sigma^{*}$ into $S$ are called (formal) 
power series. The values of $r$ are denoted by $(r, w)$, where $w \in \Sigma^{*}$, and $r$ itself is written as a formal sum

$$
r=\sum_{w \in \Sigma^{*}}(r, w) w
$$

The values $(r, w)$ are also referred to as the coefficients of the series. The collection of all power series $r$ as defined above is denoted by $S\left\langle\left\langle\Sigma^{*}\right\rangle\right\rangle$.

This terminology reflects the intuitive ideas connected with power series. We call the power series "formal" to indicate that we are not interested in summing up the series but rather, for instance, in various operations defined for series.

Given $r \in S\left\langle\left\langle\Sigma^{*}\right\rangle\right\rangle$, the support of $r$ is the set

$$
\operatorname{supp}(r)=\left\{w \in \Sigma^{*} \mid(r, w) \neq 0\right\} .
$$

A series $r \in S\left\langle\left\langle\Sigma^{*}\right\rangle\right\rangle$ where every coefficient equals 0 or 1 is termed the characteristic series of its support $L$, in symbols, $r=\operatorname{char}(L)$ or $r=\mathbb{1}_{L}$. The subset of $S\left\langle\left\langle\Sigma^{*}\right\rangle\right\rangle$ consisting of all series with a finite support is denoted by $S\left\langle\Sigma^{*}\right\rangle$. Series of $S\left\langle\Sigma^{*}\right\rangle$ are referred to as polynomials. It will be convenient to use the notations $S\langle\Sigma \cup\{\varepsilon\}\rangle, S\langle\Sigma\rangle$ and $S\langle\varepsilon\rangle$ for the collection of polynomials having their supports in $\Sigma \cup\{\varepsilon\}, \Sigma$ and $\{\varepsilon\}$, respectively.

Examples of polynomials belonging to $S\left\langle\Sigma^{*}\right\rangle$ are 0 and $a w$, where $a \in S$ and $w \in \Sigma^{*}$, defined by:

$$
\begin{aligned}
& (0, w)=0 \text { for all } w \\
& (a w, w)=a \text { and }\left(a w, w^{\prime}\right)=0 \text { for } w \neq w^{\prime} .
\end{aligned}
$$

Often $1 w$ is denoted by $w$ or $\mathbb{1}_{\{w\}}$.

We now introduce several operations on power series. For $r_{1}, r_{2}, r \in S\left\langle\left\langle\Sigma^{*}\right\rangle\right\rangle$ and $s \in S$ we define the sum $r_{1}+r_{2}$, the (Cauchy) product $r_{1} \cdot r_{2}$, the Hadamard product $r_{1} \odot r_{2}$ and scalar products $s r, r s$, each as a series belonging to $S\left\langle\left\langle\Sigma^{*}\right\rangle\right\rangle$, as follows:

- $\left(r_{1}+r_{2}, w\right)=\left(r_{1}, w\right)+\left(r_{2}, w\right)$

- $\left(r_{1} \cdot r_{2}, w\right)=\sum_{w_{1} w_{2}=w}\left(r_{1}, w_{1}\right)\left(r_{2}, w_{2}\right)$

- $\left(r_{1} \odot r_{2}, w\right)=\left(r_{1}, w\right)\left(r_{2}, w\right)$

- $(s r, w)=s(r, w)$

- $(r s, w)=(r, w) s$

for all $w \in \Sigma^{*}$.

It can be checked that $\left\langle S\left\langle\left\langle\Sigma^{*}\right\rangle\right\rangle,+, \cdot, 0, \varepsilon\right\rangle$ and $\left\langle S\left\langle\Sigma^{*}\right\rangle,+, \cdot, 0, \varepsilon\right\rangle$ are semirings, the semirings of formal power series resp. of polynomials over $\Sigma$ and $S$.

We just note that the structure $\left\langle S\left\langle\left\langle\Sigma^{*}\right\rangle\right\rangle,+, \odot, 0, \operatorname{char}\left(\Sigma^{*}\right)\right\rangle$ is also a semiring (the full Cartesian product of $\Sigma^{*}$ copies of the semiring $\langle S,+, \cdot, 0,1\rangle$ ).

Clearly, the formal language semiring $\left\langle 2^{\Sigma^{*}}, \cup, \cdot, \emptyset,\{\varepsilon\}\right\rangle$ is isomorphic to $\left\langle\mathbb{B}\left\langle\left\langle\Sigma^{*}\right\rangle\right\rangle,+, \cdot, 0, \varepsilon\right\rangle$. Essentially, a transition from $2^{\Sigma^{*}}$ to $\mathbb{B}\left\langle\left\langle\Sigma^{*}\right\rangle\right\rangle$ and vice versa 
means a transition from $L$ to $\operatorname{char}(L)$ and from $r$ to $\operatorname{supp}(r)$, respectively. Furthermore, the operation corresponding to the Hadamard product is the intersection of languages. If $r_{1}$ and $r_{2}$ are the characteristic series of the languages $L_{1}$ and $L_{2}$, then $r_{1} \odot r_{2}$ is the characteristic series of $L_{1} \cap L_{2}$.

This basic transition between $2^{\Sigma^{*}}$ and $\mathbb{B}\left\langle\left\langle\Sigma^{*}\right\rangle\right\rangle$ will be very important in all of the following as it often gives a hint how to generalize classical results from formal language theory into the realm of formal power series (with an arbitrary or suitable semiring $S$ replacing $\mathbb{B}$ ).

Let $r_{i} \in S\left\langle\left\langle\Sigma^{*}\right\rangle\right\rangle(i \in I)$, where $I$ is an arbitrary index set. Then, for $w \in \Sigma^{*}$ let $I_{w}=\left\{i \mid\left(r_{i}, w\right) \neq 0\right\}$. Assume now that for all $w \in \Sigma^{*}, I_{w}$ is finite. Then we call the family of power series $\left\{r_{i} \mid i \in I\right\}$ locally finite. In this case we can define the sum $\sum_{i \in I} r_{i}$ by

$$
\left(\sum_{i \in I} r_{i}, w\right)=\sum_{i \in I_{w}}\left(r_{i}, w\right)
$$

for all $w \in \Sigma^{*}$. Also, in this case for each $r \in S\left\langle\left\langle\Sigma^{*}\right\rangle\right\rangle$, the families $\left\{r \cdot r_{i} \mid i \in I\right\}$ and $\left\{r_{i} \cdot r \mid i \in I\right\}$ are also locally finite, and $r \cdot \sum_{i \in I} r_{i}=\sum_{i \in I} r \cdot r_{i}$ and $\left(\sum_{i \in I} r_{i}\right) \cdot r=\sum_{i \in I} r_{i} \cdot r$. Indeed, let $w \in \Sigma^{*}$ and put $J=\bigcup_{w=u v} I_{v}$, a finite set. Then

$$
\begin{aligned}
\left(r \cdot \sum_{i \in I} r_{i}, w\right) & =\sum_{w=u v}(r, u)\left(\sum_{i \in J} r_{i}, v\right)=\sum_{w=u v} \sum_{i \in J}(r, u) \cdot\left(r_{i}, v\right) \\
& =\sum_{i \in J} \sum_{w=u v}(r, u) \cdot\left(r_{i}, v\right)=\sum_{i \in J}\left(r \cdot r_{i}, w\right)=\left(\sum_{i \in I} r \cdot r_{i}, w\right),
\end{aligned}
$$

as $\left(r \cdot r_{i}, w\right) \neq 0$ implies $i \in J$. This proves the first equation, and the second one follows similarly.

A power series $r \in S\left\langle\left\langle\Sigma^{*}\right\rangle\right\rangle$ is called proper or quasiregular if $(r, \varepsilon)=0$. The star $r^{*}$ of a proper power series $r \in S\left\langle\left\langle\Sigma^{*}\right\rangle\right\rangle$ is defined by

$$
r^{*}=\sum_{n \geq 0} r^{n}
$$

Since $r$ is proper we infer $\left(r^{n}, w\right)=0$ for each $n>|w|$. Hence, $\left\{r^{n} \mid n \geq 0\right\}$ is locally finite, $\left(r^{*}, w\right)=\sum_{0 \leq n \leq|w|}\left(r^{n}, w\right)$, and the star of a proper power series is well-defined.

We generalize this result to cycle-free power series. A power series $r \in$ $S\left\langle\left\langle\Sigma^{*}\right\rangle\right\rangle$ is called cycle-free of index $k>0$ if $(r, \varepsilon)^{k}=0$. It is called cycle-free if there exists a $k \geq 1$ such that $r$ is cycle-free of index $k$. Again, we define the star of a cycle-free power series $r \in S\left\langle\left\langle\Sigma^{*}\right\rangle\right\rangle$ by

$$
r^{*}=\sum_{n \geq 0} r^{n}
$$

Since $r$ is cycle-free of some index $k \geq 1$, an easy proof by induction on the length of $w \in \Sigma^{*}$ yields $\left(r^{n}, w\right)=0$ for $n \geq k \cdot(|w|+1)$. Hence, $\left\{r^{n} \mid n \geq 0\right\}$ 
is locally finite, $\left(r^{*}, w\right)=\sum_{0 \leq n<k(|w|+1)}\left(r^{n}, w\right)$, and the star of a cycle-free power series is well-defined.

Next we wish to consider identities that are valid for a cycle-free power series $r$, like, e.g., $r r^{*}+\varepsilon=r^{*} r+\varepsilon=r^{*}$. Using the distributivity laws given above for locally finite families, this follows from:

$$
r r^{*}+\varepsilon=r \cdot \sum_{n \geq 0} r^{n}+\varepsilon=\sum_{n \geq 0} r^{n+1}+\varepsilon=r^{*} .
$$

Theorem 3.1. Let $r$ be a cycle-free power series. Then, for each $n \geq 0$,

$$
r^{*}=r^{n+1} r^{*}+\sum_{0 \leq j \leq n} r^{j}=r^{*} r^{n+1}+\sum_{0 \leq j \leq n} r^{j} .
$$

Proof. We obtain by substitutions

$$
r^{*}=r r^{*}+\varepsilon=r\left(r r^{*}+\varepsilon\right)+\varepsilon=r^{2} r^{*}+r+\varepsilon=\ldots .
$$

The proof of the second equality is analogous.

Theorem 3.2. Let $r, r^{\prime} \in S\left\langle\left\langle\Sigma^{*}\right\rangle\right\rangle$ and assume that $r r^{\prime}$ is cycle-free. Then $r^{\prime} r$ is cycle-free and

$$
\left(r r^{\prime}\right)^{*} r=r\left(r^{\prime} r\right)^{*}
$$

Proof. Since $r r^{\prime}$ is cycle-free, $\left(\left(r r^{\prime}\right)^{k}, \varepsilon\right)=0$ for some $k>0$. Hence,

$$
\left(\left(r^{\prime} r\right)^{k+1}, \varepsilon\right)=\left(r^{\prime}, \varepsilon\right)\left(\left(r r^{\prime}\right)^{k}, \varepsilon\right)(r, \varepsilon)=0
$$

and $r^{\prime} r$ is cycle-free. It follows that $\left(r r^{\prime}\right)^{*} r=\sum_{n \geq 0}\left(\left(r r^{\prime}\right)^{n} \cdot r\right)=\sum_{n \geq 0} r$. $\left(r^{\prime} r\right)^{n}=r \cdot\left(r^{\prime} r\right)^{*}$.

The Hurwitz product (also called shuffle product) is defined as follows. For $w_{1}, w_{2} \in \Sigma^{*}$ and $x_{1}, x_{2} \in \Sigma$, we define $w_{1} \sqcup w_{2} \in S\left\langle\left\langle\Sigma^{*}\right\rangle\right\rangle$ by

$$
w_{1} \sqcup \varepsilon=w_{1}, \quad \varepsilon \sqcup w_{2}=w_{2},
$$

and

$$
w_{1} x_{1} \sqcup w_{2} x_{2}=\left(w_{1} x_{1} \sqcup w_{2}\right) x_{2}+\left(w_{1} \sqcup w_{2} x_{2}\right) x_{1} .
$$

For $r_{1}, r_{2} \in S\left\langle\left\langle\Sigma^{*}\right\rangle\right\rangle$, the Hurwitz product $r_{1} \sqcup r_{2} \in S\left\langle\left\langle\Sigma^{*}\right\rangle\right\rangle$ of $r_{1}$ and $r_{2}$ is then defined by

$$
r_{1} \sqcup r_{2}=\sum_{w_{1}, w_{2} \in \Sigma^{*}}\left(r_{1}, w_{1}\right)\left(r_{2}, w_{2}\right)\left(w_{1} \sqcup w_{2}\right) .
$$

Observe that

$$
\left(r_{1} \sqcup r_{2}, w\right)=\sum_{\left|w_{1}\right|+\left|w_{2}\right|=|w|}\left(r_{1}, w_{1}\right)\left(r_{2}, w_{2}\right)\left(w_{1} \sqcup w_{2}, w\right)
$$


is a finite sum for all $w \in \Sigma^{*}$. Hence, $\left\{\sum_{w_{1} w_{2}=w}\left(r_{1}, w_{1}\right)\left(r_{2}, w_{2}\right) w_{1} \amalg w_{2} \mid\right.$ $\left.w \in \Sigma^{*}\right\}$ is locally finite and the Hurwitz product of two power series is well-defined.

In language theory, the shuffle product is customarily defined for languages $L$ and $L^{\prime}$ by

$$
L \sqcup L^{\prime}=\left\{w_{1} w_{1}^{\prime} \ldots w_{n} w_{n}^{\prime} \mid w_{1} \ldots w_{n} \in L, w_{1}^{\prime} \ldots w_{n}^{\prime} \in L^{\prime}, n \geq 1\right\} .
$$

If $r_{1}, r_{2} \in \mathbb{B}\left\langle\left\langle\Sigma^{*}\right\rangle\right\rangle$ then this definition is "isomorphic" to that given above for formal power series.

When the semiring $S$ is ordered by $\leq$, we may order $S\left\langle\left\langle\Sigma^{*}\right\rangle\right\rangle$, and thus $S\left\langle\Sigma^{*}\right\rangle$ by the pointwise order: We define $r \leq r^{\prime}$ for $r, r^{\prime} \in S\left\langle\left\langle\Sigma^{*}\right\rangle\right\rangle$ iff $(r, w) \leq$ $\left(r^{\prime}, w\right)$ for all $w \in \Sigma^{*}$. Equipped with this order, clearly both $S\left\langle\left\langle\Sigma^{*}\right\rangle\right\rangle$ and $S\left\langle\Sigma^{*}\right\rangle$ are ordered semirings.

If $\langle S,+, \cdot, 0,1\rangle$ is a complete semiring, we can define an infinitary sum operation on $S\left\langle\left\langle\Sigma^{*}\right\rangle\right\rangle$ as follows: If $r_{i} \in S\left\langle\left\langle\Sigma^{*}\right\rangle\right\rangle$ for $i \in I$, then $\sum_{i \in I} r_{i}=$ $\sum_{w \in \Sigma^{*}}\left(\sum_{i \in I}\left(r_{i}, w\right)\right) w$. We obtain:

Proposition 3.3. Let $S$ be a semiring.

(a) If $S$ is complete, $S\left\langle\left\langle\Sigma^{*}\right\rangle\right\rangle$ is also complete.

(b) If $S$ is continuous, $S\left\langle\left\langle\Sigma^{*}\right\rangle\right\rangle$ is also continuous.

(c) If $S$ is a Conway semiring, then $S\left\langle\left\langle\Sigma^{*}\right\rangle\right\rangle$ is also a Conway semiring.

Here (a) and (b) follow straightforwardly by arguing element-wise for each word $w \in \Sigma^{*}$, and for part (c) see Bloom, Ésik [4] and Ésik, Kuich [10].

Proposition 3.3 and the Hurwitz product are now used to prove that each complete star semiring is a Conway semiring (see Kuich [28], Hebisch [20]).

Theorem 3.4. Each complete star semiring is a Conway semiring.

Proof. Let $S$ be a complete star semiring and let $a, b \in S$. Let $\bar{a}, \bar{b}$ be letters. Note that to each word $\bar{w}=\overline{c_{1}} \overline{c_{2}} \ldots \overline{c_{n}}$, with $\overline{c_{i}} \in\{\bar{a}, \bar{b}\}$ for $1 \leq i \leq n$, there corresponds the element $w=c_{1} c_{2} \ldots c_{n} \in S$. By Proposition 3.3, $\left\langle S\left\langle\left\langle\Sigma^{*}\right\rangle\right\rangle,+, \cdot, 0, \varepsilon\right\rangle$ is a complete semiring. Also, observe that $\bar{a} \mapsto a, \bar{b} \mapsto b$ induces a star semiring morphism from the complete star semiring $S\left\langle\left\langle\{\bar{a}, \bar{b}\}^{*}\right\rangle\right\rangle$ to $S$.

Using induction, the following equalities can be shown for all $n, m \geq 0$ :

$$
\begin{gathered}
(\bar{a}+\bar{b})^{n}=\sum_{0 \leq j \leq n} \bar{a}^{j} \amalg \bar{b}^{n-j}, \\
\bar{a}^{n} \amalg \bar{b}^{m}=\sum_{0 \leq j \leq n}\left(\bar{a}^{j} \amalg \bar{b}^{m-1}\right) \bar{b} \bar{a}^{n-j}
\end{gathered}
$$

and

$$
\bar{a}^{*} \sqcup \bar{b}^{n}=\sum_{j \geq 0} \bar{a}^{j} \amalg \bar{b}^{n}=\left(\bar{a}^{*} \bar{b}\right)^{n} \bar{a}^{*} .
$$


Hence, we infer the equality

$$
(\bar{a}+\bar{b})^{*}=\sum_{n \geq 0} \sum_{j \geq 0} \bar{a}^{j} \sqcup \bar{b}^{n},
$$

which implies immediately

$$
(\bar{a}+\bar{b})^{*}=\left(\bar{a}^{*} \bar{b}\right)^{*} \bar{a}^{*}
$$

Applying the star semiring morphism defined above we obtain the sum star identity in $S$ :

$$
(a+b)^{*}=\left(a^{*} b\right)^{*} a^{*} .
$$

The product star identity is clear by

$$
\begin{aligned}
(a b)^{*}= & 1+\sum_{n \geq 1}(a b)^{n}=1+a\left(\sum_{n \geq 0}(b a)^{n}\right) b= \\
& 1+a(b a)^{*} b .
\end{aligned}
$$

Finally, we show that morphisms between two semirings and also particular morphisms between free monoids induce morphisms between the associated semirings of formal power series.

First, let $\Sigma$ be an alphabet, $S, S^{\prime}$ two semirings and $h: S \rightarrow S^{\prime}$ a morphism. We define $\bar{h}: S\left\langle\left\langle\Sigma^{*}\right\rangle\right\rangle \rightarrow S^{\prime}\left\langle\left\langle\Sigma^{*}\right\rangle\right\rangle$ by $\bar{h}(r)=h \circ r$ for each $r \in S\left\langle\left\langle\Sigma^{*}\right\rangle\right\rangle$, i. e. $(\bar{h}(r), w)=h((r, w))$ for each $w \in \Sigma^{*}$. Often $\bar{h}$ is again denoted by $h$. The following is straightforward by elementary calculations.

Proposition 3.5. Let $\Sigma$ be an alphabet, $S, S^{\prime}$ two semirings and $h: S \rightarrow$ $S^{\prime}$ a semiring morphism. Then $h: S\left\langle\left\langle\Sigma^{*}\right\rangle\right\rangle \rightarrow S^{\prime}\left\langle\left\langle\Sigma^{*}\right\rangle\right\rangle$ is again a semiring morphism. Moreover, if $r$ is cycle-free, so is $h(r)$ and $h\left(r^{*}\right)=(h(r))^{*}$.

Second, let $S$ be a semiring, $\Sigma, \Sigma^{\prime}$ two alphabets and $h: \Sigma^{*} \rightarrow \Sigma^{* *}$ a morphism. We define $h^{-1}: S\left\langle\left\langle\Sigma^{\prime *}\right\rangle\right\rangle \rightarrow S\left\langle\left\langle\Sigma^{*}\right\rangle\right\rangle$ by $h^{-1}\left(r^{\prime}\right)=r^{\prime} \circ h$ for each $r^{\prime} \in S\left\langle\left\langle\Sigma^{\prime *}\right\rangle\right\rangle$, that is, $\left(h^{-1}\left(r^{\prime}\right), v\right)=\left(r^{\prime}, h(v)\right)$ for each $v \in \Sigma^{*}$. We call $h: \Sigma^{*} \rightarrow \Sigma^{\prime *}$ length-preserving, if $|v|=|h(v)|$ for each $v \in \Sigma^{*}$; equivalently, $h(x) \in \Sigma^{\prime}$ for each $x \in \Sigma$. Further, $h$ is non-deleting, if $h(x) \neq \varepsilon$ for each $x \in \Sigma$; equivalently, $|v| \leq|h(v)|$ for each $v \in \Sigma^{*}$. If $h$ is non-deleting or if $S$ is complete, we define $\bar{h}: S\left\langle\left\langle\Sigma^{*}\right\rangle\right\rangle \rightarrow S\left\langle\left\langle\Sigma^{* *}\right\rangle\right\rangle$ by letting $(\bar{h}(r), w)=$ $\sum_{v \in \Sigma^{*}, h(v)=w}(r, v)$ for each $r \in S\left\langle\left\langle\Sigma^{*}\right\rangle\right\rangle$ and $w \in \Sigma^{* *}$. Observe that if $h$ is non-deleting, $h^{-1}(w)$ is a finite set for each $w \in \Sigma^{*}$, and hence $\bar{h}(r)$ is welldefined.

Proposition 3.6. Let $S$ be a semiring, $\Sigma, \Sigma^{\prime}$ two alphabets and $h: \Sigma^{*} \rightarrow \Sigma^{*}$ a morphism.

(i) Let $h$ be length-preserving. Then the mapping $h^{-1}: S\left\langle\left\langle\Sigma^{* *}\right\rangle\right\rangle \rightarrow S\left\langle\left\langle\Sigma^{*}\right\rangle\right\rangle$ is a semiring morphism. Moreover, if $r^{\prime} \in S\left\langle\left\langle\Sigma^{\prime *}\right\rangle\right\rangle$ is cycle-free then so is $h^{-1}\left(r^{\prime}\right)$, and $h^{-1}\left(r^{*}\right)=\left(h^{-1}\left(r^{\prime}\right)\right)^{*}$ 
(ii) Let $h$ be non-deleting, or assume that $S$ is complete. Then $\bar{h}: S\left\langle\left\langle\Sigma^{*}\right\rangle\right\rangle \rightarrow$ $S\left\langle\left\langle\Sigma^{\prime *}\right\rangle\right\rangle$ is a semiring morphism. Moreover, if $h$ is non-deleting and $r \in$ $S\left\langle\left\langle\Sigma^{*}\right\rangle\right\rangle$ is cycle-free then so is $\bar{h}(r)$, and $\bar{h}\left(r^{*}\right)=(\bar{h}(r))^{*}$.

Proof. Again, by elementary calculations. For (i), note that if $v \in \Sigma^{*}$ and $h(v)=w_{1} w_{2}$ with $w_{1}, w_{2} \in \Sigma^{\prime *}$, then since $h$ is length-preserving, there are $v_{1}, v_{2} \in \Sigma^{*}$ with $v=v_{1} v_{2}$ and $h\left(v_{1}\right)=w_{1}, h\left(v_{2}\right)=w_{2}$. This implies that $h^{-1}$ preserves the Cauchy product.

\section{Matrices}

We now introduce (possibly infinite) matrices. These are important here since the structure and the behaviour of weighted automata can often be compactly described using matrices (see chapters $[13,35,39]$ ) and hence results from matrix algebra can be used to derive results for weighted automata.

Consider two non-empty index sets $I$ and $I^{\prime}$ and a set $U$. A mapping $A: I \times I^{\prime} \rightarrow U$ is called a matrix. The values of $A$ are denoted by $A_{i, i^{\prime}}$, where $i \in I$ and $i^{\prime} \in I^{\prime}$. The values $A_{i, i^{\prime}}$ are also referred to as the entries of the matrix $A$. In particular, $A_{i, i^{\prime}}$ is called the $\left(i, i^{\prime}\right)$-entry of $A$. The collection of all matrices as defined above is denoted by $U^{I \times I^{\prime}}$.

If both $I$ and $I^{\prime}$ are finite, then $A$ is called a finite matrix. If $I$ or $I^{\prime}$ is a singleton, then $A^{I \times I^{\prime}}$ is denoted by $A^{1 \times I^{\prime}}$ or $A^{I \times 1}$, and $A$ is called a row or column vector, respectively. If $A \in U^{I \times 1}$ (resp. $A \in U^{1 \times I^{\prime}}$ ) then we often denote the $i$-th entry of $A$ for $i \in I$ (resp. $i \in I^{\prime}$ ), by $A_{i}$ instead of $A_{i, 1}$ (resp. $\left.A_{1, i}\right)$. If $I=\{1, \ldots, m\}$ and $I^{\prime}=\{1, \ldots, n\}$, the set $U^{I \times I^{\prime}}$ is denoted by $U^{m \times n}$.

As before we introduce some operations and special matrices inducing a monoid or semiring structure to matrices. Let $S$ be a semiring. For $A, B \in$ $S^{I \times I^{\prime}}$ we define the sum $A+B \in S^{I \times I^{\prime}}$ by $(A+B)_{i, i^{\prime}}=A_{i, i^{\prime}}+B_{i, i^{\prime}}$ for all $i \in I, i^{\prime} \in I^{\prime}$. Furthermore, we introduce the zero matrix $0 \in S^{I \times I^{\prime}}$. All entries of the zero matrix 0 are 0 . By these definitions, $\left\langle S^{I \times I^{\prime}},+, 0\right\rangle$ is a commutative monoid.

Let $A \in S^{I \times I^{\prime}}$. Consider, for $i \in I$, the set of indices $\left\{j \mid A_{i j} \neq 0\right\}$. Then $A$ is called a row finite matrix if these sets are finite for all $i \in I$. Similarly, consider, for $i^{\prime} \in I^{\prime}$, the set of indices $\left\{j \mid A_{j i^{\prime}} \neq 0\right\}$. Then $A$ is called a column finite matrix if these sets are finite for all $i^{\prime} \in I^{\prime}$.

If $A$ is row finite or $B$ is column finite, or if $S$ is complete, then, for $A \in S^{I_{1} \times I_{2}}$ and $B \in S^{I_{2} \times I_{3}}$, we define the product $A B \in S^{I_{1} \times I_{3}}$ by

$$
(A B)_{i_{1}, i_{3}}=\sum_{i_{2} \in I_{2}} A_{i_{1}, i_{2}} B_{i_{2}, i_{3}} \quad \text { for all } i_{1} \in I_{1}, i_{3} \in I_{3} .
$$

Furthermore, we introduce the matrix of unity $E \in S^{I \times I}$. The diagonal entries $E_{i, i}$ of $E$ are equal to 1, the off-diagonal entries $E_{i_{1}, i_{2}}\left(i_{1} \neq i_{2}\right)$ of $E$ are equal to 0 , for $i, i_{1}, i_{2} \in I$. 
It is easily shown that matrix multiplication is associative, the distributivity laws are valid for matrix addition and multiplication, $E$ is a multiplicative unit and 0 is a multiplicative zero. So we infer that $\left\langle S^{I \times I},+, \cdot, 0, E\right\rangle$ is a semiring if $I$ is finite or if $S$ is complete. Moreover, the row finite matrices in $S^{I \times I}$ and the column finite matrices in $S^{I \times I}$ form semirings.

If $S$ is complete, infinite sums can be extended to matrices. Consider $S^{I \times I^{\prime}}$ and define, for $A_{j} \in S^{I \times I^{\prime}}, j \in J$, where $J$ is an index set, $\sum_{j \in J} A_{j}$ by its entries:

$$
\left(\sum_{j \in J} A_{j}\right)_{i, i^{\prime}}=\sum_{j \in J}\left(A_{j}\right)_{i, i^{\prime}}, \quad \text { for all } i \in I, i^{\prime} \in I^{\prime} .
$$

By this definition, $S^{I \times I}$ is a complete semiring.

If $S$ is ordered, the order on $S$ is extended pointwise to matrices $A$ and $B$ in $S^{I \times I^{\prime}}$ :

$$
A \leq B \quad \text { if } \quad A_{i, i^{\prime}} \leq B_{i, i^{\prime}} \text { for all } i \in I, i^{\prime} \in I^{\prime} .
$$

If $S$ is continuous then so is $S^{I \times I}$.

Eventually, if $S$ is a locally closed star semiring then $S^{n \times n}, n \geq 1$, is again a locally closed star semiring (see Ésik, Kuich [11], Zhao [42]); and if $S$ is a Conway semiring then $S^{n \times n}, n \geq 1$, is again a Conway semiring (see Conway [6], Bloom, Ésik [4], Ésik, Kuich [10]). Clearly, if $S$ is locally finite, then so is $S^{n \times n}$ for each $n \geq 1$ (cf. [7]).

For the remainder of this chapter, $I$ (resp. $Q$ ), possibly provided with indices, denotes an arbitrary (resp. finite) index set. For the rest of this section we assume that all products of matrices are well-defined.

We now introduce blocks of matrices. Consider a matrix $A$ in $S^{I \times I}$. Assume that we have a decomposition $I=\bigcup_{j \in J} I_{j}$ where $J$ and all $I_{j}(j \in J)$ are non-empty index sets such that $I_{j_{1}} \cap I_{j_{2}}=\emptyset$ for $j_{1} \neq j_{2}$. The mapping $A$, restricted to the domain $I_{j_{1}} \times I_{j_{2}}$, i. e., $A \uparrow_{I_{j_{1}} \times I_{j_{2}}}: I_{j_{1}} \times I_{j_{2}} \rightarrow S$ is, of course, a matrix in $S^{I_{j_{1}} \times I_{j_{2}}}$. We denote it by $A\left(I_{j_{1}}, I_{j_{2}}\right)$ and call it the $\left(I_{j_{1}}, I_{j_{2}}\right)$-block of $A$.

We can compute the blocks of the sum and the product of matrices $A$ and $B$ from the blocks of $A$ and $B$ in the usual way:

$$
\begin{aligned}
(A+B)\left(I_{j_{1}}, I_{j_{2}}\right) & =A\left(I_{j_{1}}, I_{j_{2}}\right)+B\left(I_{j_{1}}, I_{j_{2}}\right) \\
(A B)\left(I_{j_{1}}, I_{j_{2}}\right) & =\sum_{j \in J} A\left(I_{j_{1}}, I_{j}\right) B\left(I_{j}, I_{j_{2}}\right) .
\end{aligned}
$$

In a similar manner, the matrices of $S^{I \times I^{\prime}}$ can be partitioned into blocks. This yields the computational rule

$$
(A+B)\left(I_{j}, I_{j^{\prime}}^{\prime}\right)=A\left(I_{j}, I_{j^{\prime}}^{\prime}\right)+B\left(I_{j}, I_{j^{\prime}}^{\prime}\right)
$$

If we consider matrices $A \in S^{I \times I^{\prime}}$ and $B \in S^{I^{\prime} \times I^{\prime \prime}}$ partitioned into compatible blocks, i. e., $I^{\prime}$ is partitioned into the same index sets for both matrices, then we obtain the computational rule 


$$
(A B)\left(I_{j}, I_{j^{\prime \prime}}^{\prime \prime}\right)=\sum_{j^{\prime} \in J^{\prime}} A\left(I_{j}, I_{j^{\prime}}^{\prime}\right) B\left(I_{j^{\prime}}^{\prime}, I_{j^{\prime \prime}}^{\prime \prime}\right) \text {. }
$$

Now let us assume that $I$ and $I^{\prime}$ are finite, or that $S$ is complete. In the sequel the following isomorphisms are needed:

(i) The semirings

$$
\left(S^{I^{\prime} \times I^{\prime}}\right)^{I \times I}, S^{\left(I \times I^{\prime}\right) \times\left(I \times I^{\prime}\right)}, S^{\left(I^{\prime} \times I\right) \times\left(I^{\prime} \times I\right)},\left(S^{I \times I}\right)^{I^{\prime} \times I^{\prime}}
$$

are isomorphic by the correspondences between

$$
\left(A_{i_{1}, i_{2}}\right)_{i_{1}^{\prime}, i_{2}^{\prime}}, A_{\left(i_{1}, i_{1}^{\prime}\right),\left(i_{2}, i_{2}^{\prime}\right)}, A_{\left(i_{1}^{\prime}, i_{1}\right),\left(i_{2}^{\prime}, i_{2}\right)},\left(A_{i_{1}^{\prime}, i_{2}^{\prime}}\right)_{i_{1}, i_{2}}
$$

for all $i_{1}, i_{2} \in I, i_{1}^{\prime}, i_{2}^{\prime} \in I^{\prime}$.

(ii) The semirings $S^{I \times I}\left\langle\left\langle\Sigma^{*}\right\rangle\right\rangle$ and $\left(S\left\langle\left\langle\Sigma^{*}\right\rangle\right\rangle\right)^{I \times I}$ are isomorphic by the correspondence between $(A, w)_{i_{1}, i_{2}}$ and $\left(A_{i_{1}, i_{2}}, w\right)$ for all $i_{1}, i_{2} \in I, w \in \Sigma^{*}$.

Moreover, analogous isomorphisms are valid if the semirings of row finite or column finite matrices are considered. Observe that, in case $S$ is complete, these correspondences are isomorphisms of complete semirings, i. e., they respect infinite sums. These isomorphisms are used without further mention. Moreover, the notation $A_{i_{1}, i_{2}}$, where $A \in S^{I_{1} \times I_{2}}\left\langle\left\langle\Sigma^{*}\right\rangle\right\rangle$ and $i_{1} \in I_{1}, i_{2} \in I_{2}$, is used: $A_{i_{1}, i_{2}}$ is the power series in $S\left\langle\left\langle\Sigma^{*}\right\rangle\right\rangle$ such that the coefficient $\left(A_{i_{1}, i_{2}}, w\right)$ of $w \in \Sigma^{*}$ is equal to $(A, w)_{i_{1}, i_{2}}$. Similarly, the notation $(A, w)$, where $A \in\left(S\left\langle\left\langle\Sigma^{*}\right\rangle\right\rangle\right)^{I_{1} \times I_{2}}$ and $w \in \Sigma^{*}$, is used: $(A, w)$ is the matrix in $S^{I_{1} \times I_{2}}$ whose $\left(i_{1}, i_{2}\right)$-entry $(A, w)_{i_{1}, i_{2}}$ is equal to $\left(A_{i_{1}, i_{2}}, w\right)$ for each $i_{1} \in I_{1}, i_{2} \in I_{2}$.

For the proof of the next theorem we need a lemma.

Lemma 4.1. Let $S$ be a complete star semiring. Then, for all $s_{1}, s_{2} \in S$,

$$
\left(s_{1}+s_{2}\right)^{*}=\left(s_{1}+s_{2} s_{1}^{*} s_{2}\right)^{*}\left(1+s_{2} s_{1}^{*}\right) .
$$

Proof. Using Theorem 3.4, we have $\left(s_{1}+s_{2} s_{1}^{*} s_{2}\right)^{*}\left(1+s_{2} s_{1}^{*}\right)=\left(s_{1}^{*} s_{2} s_{1}^{*} s_{2}\right)^{*} s_{1}^{*}(1+$ $\left.s_{2} s_{1}^{*}\right)=\sum_{n \geq 0}\left(s_{1}^{*} s_{2}\right)^{2 n} s_{1}^{*}+\sum_{n \geq 0}\left(s_{1}^{*} s_{2}\right)^{2 n+1} s_{1}^{*}=\left(s_{1}^{*} s_{2}\right)^{*} s_{1}^{*}=\left(s_{1}+s_{2}\right)^{*}$.

The next theorem is central for automata theory (see Conway [6], Lehmann [31], Kuich, Salomaa [30], Kuich [29], Bloom, Ésik [4], Kozen [25]). It allows us to compute the blocks of the star of a matrix $A$ by sum, product and star of the blocks of $A$.

For notational convenience, we will denote in Theorem 4.2 and in Corollaries 4.3 and 4.4 the matrices $A\left(I_{i}, I_{j}\right)$ by $A_{i, j}$, for $1 \leq i, j \leq 3$.

Theorem 4.2. Let $S$ be a complete star semiring. Let $A \in S^{I \times I}$ and $I=$ $I_{1} \cup I_{2}$ with $I_{1}, I_{2} \neq \emptyset$ and $I_{1} \cap I_{2}=\emptyset$. Then

$$
A^{*}\left(I_{1}, I_{1}\right)=\left(A_{1,1}+A_{1,2} A_{2,2}^{*} A_{2,1}\right)^{*},
$$




$$
\begin{aligned}
& A^{*}\left(I_{1}, I_{2}\right)=\left(A_{1,1}+A_{1,2} A_{2,2}^{*} A_{2,1}\right)^{*} A_{1,2} A_{2,2}^{*}, \\
& A^{*}\left(I_{2}, I_{1}\right)=\left(A_{2,2}+A_{2,1} A_{1,1}^{*} A_{1,2}\right)^{*} A_{2,1} A_{1,1}^{*}, \\
& A^{*}\left(I_{2}, I_{2}\right)=\left(A_{2,2}+A_{2,1} A_{1,1}^{*} A_{1,2}\right)^{*} .
\end{aligned}
$$

Proof. Consider the matrices

$$
A_{1}=\left(\begin{array}{cc}
A_{1,1} & 0 \\
0 & A_{2,2}
\end{array}\right) \quad \text { and } \quad A_{2}=\left(\begin{array}{cc}
0 & A_{1,2} \\
A_{2,1} & 0
\end{array}\right) .
$$

The computation of $\left(A_{1}+A_{2} A_{1}^{*} A_{2}\right)^{*}\left(E+A_{2} A_{1}^{*}\right)$ and application of Lemma 4.1 prove our theorem.

Corollary 4.3. If $A_{2,1}=0$ then

$$
A^{*}=\left(\begin{array}{cc}
A_{1,1}^{*} & A_{1,1}^{*} A_{1,2} A_{2,2}^{*} \\
0 & A_{2,2}^{*}
\end{array}\right) .
$$

Corollary 4.4. Let $I=I_{1} \cup I_{2} \cup I_{3}$ be a decomposition into pairwise disjoint non-empty subsets. If $A_{2,1}=0, A_{3,1}=0$ and $A_{3,2}=0$ then

$$
A^{*}=\left(\begin{array}{ccc}
A_{1,1}^{*} & A_{1,1}^{*} A_{1,2} A_{2,2}^{*} & A_{1,1}^{*} A_{1,2} A_{2,2}^{*} A_{2,3} A_{3,3}^{*}+A_{1,1}^{*} A_{1,3} A_{3,3}^{*} \\
0 & A_{2,2}^{*} & A_{2,2}^{*} A_{2,3} A_{3,3}^{*} \\
0 & 0 & A_{3,3}^{*}
\end{array}\right)
$$

Next we consider an arbitrary partition of the index set $I$.

Theorem 4.5. Let $S$ be a complete star semiring, and let $I=\bigcup_{j \in J} I_{j}$ be a decomposition into pairwise disjoint non-empty subsets. Fix $j_{0} \in J$. Assume that the only non-null blocks of the matrix $A \in S^{I \times I}$ are $A\left(I_{j}, I_{j_{0}}\right), A\left(I_{j_{0}}, I_{j}\right)$ and $A\left(I_{j}, I_{j}\right)$, for all $j \in J$. Then

$$
A^{*}\left(I_{j_{0}}, I_{j_{0}}\right)=\left(A\left(I_{j_{0}}, I_{j_{0}}\right)+\sum_{j \in J, j \neq j_{0}} A\left(I_{j_{0}}, I_{j}\right) A\left(I_{j}, I_{j}\right)^{*} A\left(I_{j}, I_{j_{0}}\right)\right)^{*} .
$$

Proof. We partition $I$ into $I_{j_{0}}$ and $I^{\prime}=I-I_{j_{0}}$. Then $A\left(I^{\prime}, I^{\prime}\right)$ is a blockdiagonal matrix and $\left(A\left(I^{\prime}, I^{\prime}\right)^{*}\right)\left(I_{j}, I_{j}\right)=A\left(I_{j}, I_{j}\right)^{*}$ for all $j \in J-\left\{j_{0}\right\}$. By Theorem 4.2 we obtain

$$
A^{*}\left(I_{j_{0}}, I_{j_{0}}\right)=\left(A\left(I_{j_{0}}, I_{j_{0}}\right)+A\left(I_{j_{0}}, I^{\prime}\right) A\left(I^{\prime}, I^{\prime}\right)^{*} A\left(I^{\prime}, I_{j_{0}}\right)\right)^{*} .
$$

The computation of the right side of this equality proves our theorem.

We now introduce the Kronecker product (also called tensor product) $A \otimes$ $B \in S^{\left(I_{1} \times I_{2}\right) \times\left(I_{1}^{\prime} \times I_{2}^{\prime}\right)}$ for the matrices $A \in S^{I_{1} \times I_{1}^{\prime}}$ and $B \in S^{I_{2} \times I_{2}^{\prime}}$, by defining its entries:

$(A \otimes B)_{\left(i_{1}, i_{2}\right),\left(i_{1}^{\prime}, i_{2}^{\prime}\right)}=A_{i_{1}, i_{1}^{\prime}} B_{i_{2}, i_{2}^{\prime}}$, for all $i_{1} \in I_{1}, i_{1}^{\prime} \in I_{1}^{\prime}, i_{2} \in I_{2}, i_{2}^{\prime} \in I_{2}^{\prime}$. 
Sometimes, the Kronecker product $A \otimes B$ is defined to be in $\left(S^{I_{2} \times I_{2}^{\prime}}\right)^{I_{1} \times I_{1}^{\prime}}$ with

$$
\left((A \otimes B)_{i_{1}, i_{1}^{\prime}}\right)_{i_{2}, i_{2}^{\prime}}=A_{i_{1}, i_{1}^{\prime}} B_{i_{2}, i_{2}^{\prime}}, \quad \text { for all } i_{1} \in I_{1}, i_{1}^{\prime} \in I_{1}^{\prime}, i_{2} \in I_{2}, i_{2}^{\prime} \in I_{2}^{\prime} .
$$

Since the semirings $S^{\left(I_{1} \times I_{2}\right) \times\left(I_{1}^{\prime} \times I_{2}^{\prime}\right)}$ and $\left(S^{I_{2} \times I_{2}^{\prime}}\right)^{I_{1} \times I_{1}^{\prime}}$ are isomorphic, this will not make any difference in the computations.

Easy proofs show the following computational rules for Kronecker products.

Theorem 4.6. Let $A, A^{\prime} \in S^{I_{1} \times I_{1}^{\prime}}, B, B^{\prime} \in S^{I_{2} \times I_{2}^{\prime}}, C \in S^{I_{3} \times I_{3}^{\prime}}$. Then

$$
\begin{aligned}
& \text { (i) }\left(A+A^{\prime}\right) \otimes B=A \otimes B+A^{\prime} \otimes B, \\
& \text { (ii) } A \otimes\left(B+B^{\prime}\right)=A \otimes B+A \otimes B^{\prime}, \\
& \text { (iii) } A \otimes 0=0 \text { and } 0 \otimes B=0, \\
& \text { (iv) } A \otimes(B \otimes C)=(A \otimes B) \otimes C \text {. }
\end{aligned}
$$

Theorem 4.7. Let $A \in S^{I_{1} \times I_{2}}\langle\varepsilon\rangle, B \in S^{I_{2} \times I_{3}}\langle\varepsilon\rangle, C \in S^{I_{4} \times I_{5}}\left\langle\left\langle\Sigma^{*}\right\rangle\right\rangle$ and $D \in S^{I_{5} \times I_{6}}\left\langle\left\langle\Sigma^{*}\right\rangle\right\rangle$. Assume that $S$ is complete or that $(A, \varepsilon)$ and $(C, w)$ are row finite for all $w \in \Sigma^{*}$, or that $(B, \varepsilon)$ and $(D, w)$ are column finite for all $w \in \Sigma^{*}$. Furthermore, assume that all entries of $(B, \varepsilon)$ commute with those of $(C, w)$ for all $w \in \Sigma^{*}$. Then

$$
(A B) \otimes(C D)=(A \otimes C)(B \otimes D) .
$$

Proof. Let $i_{j} \in I_{j}$ for $j=1,3,4,6$. Then we obtain

$$
\begin{aligned}
& ((A B) \otimes(C D))_{\left(i_{1}, i_{3}\right),\left(i_{4}, i_{6}\right)} \\
= & (A B)_{i_{1}, i_{3}}(C D)_{i_{4}, i_{6}} \\
= & \sum_{i_{2} \in I_{2}} \sum_{i_{5} \in I_{5}} A_{i_{1}, i_{2}} B_{i_{2}, i_{3}} C_{i_{4}, i_{5}} D_{i_{5}, i_{6}} \\
= & \sum_{i_{2} \in I_{2}} \sum_{i_{5} \in I_{5}} A_{i_{1}, i_{2}} C_{i_{4}, i_{5}} B_{i_{2}, i_{3}} D_{i_{5}, i_{6}} \\
= & \sum_{\left(i_{2}, i_{5}\right) \in I_{2} \times I_{5}}(A \otimes C)_{\left(i_{1}, i_{4}\right),\left(i_{2}, i_{5}\right)}(B \otimes D)_{\left(i_{2}, i_{5}\right),\left(i_{3}, i_{6}\right)} \\
= & ((A \otimes C)(B \otimes D))_{\left(i_{1}, i_{4}\right),\left(i_{3}, i_{6}\right)} .
\end{aligned}
$$

\section{Cycle-Free linear equations}

Let $\Sigma$ be an alphabet and $S$ any semiring. Cycle-free linear equations over $S\left\langle\left\langle\Sigma^{*}\right\rangle\right\rangle$ are a useful tool for proving identities in $S\left\langle\left\langle\Sigma^{*}\right\rangle\right\rangle$. Assume that two expressions are shown to be solutions of such an equation. Then, the uniqueness 
of the solution (shown below) implies that these two expressions represent the same formal power series in $S\left\langle\left\langle\Sigma^{*}\right\rangle\right\rangle$.

A cycle-free linear equation (over $\left.S\left\langle\left\langle\Sigma^{*}\right\rangle\right\rangle\right)$ has the form

$$
y=r y+r^{\prime},
$$

where $r, r^{\prime} \in S\left\langle\left\langle\Sigma^{*}\right\rangle\right\rangle$ and $r$ is cycle-free. A solution to this equation is given by a power series $\sigma \in S\left\langle\left\langle\Sigma^{*}\right\rangle\right\rangle$ such that $\sigma=r \sigma+r^{\prime}$.

Theorem 5.1. The cycle-free equation $y=r y+r^{\prime}$ with $r, r^{\prime} \in S\left\langle\left\langle\Sigma^{*}\right\rangle\right\rangle, r$ cycle-free, has the unique solution $\sigma=r^{*} r^{\prime}$.

Proof. By Theorem 3.1, we obtain

$$
r \sigma+r^{\prime}=r r^{*} r^{\prime}+r^{\prime}=\left(r r^{*}+\varepsilon\right) r^{\prime}=r^{*} r^{\prime}=\sigma .
$$

Hence, $\sigma$ is a solution.

Assume that $r$ is cycle-free of index $k$, i. e., $(r, \varepsilon)^{k}=0$, and that $\varrho$ is a solution. Then, by substitution, we obtain, for all $n \geq 0$,

$$
\varrho=r \varrho+r^{\prime}=\cdots=r^{n} \varrho+\sum_{0 \leq j<n} r^{j} r^{\prime} .
$$

We now compute the coefficients $(\varrho, w)$ for each $w \in \Sigma^{*}$ :

$$
(\varrho, w)=\left(r^{k(|w|+1)} \varrho, w\right)+\sum_{0 \leq j<k(|w|+1)}\left(r^{j} r^{\prime}, w\right)=\left(r^{*} r^{\prime}, w\right)=(\sigma, w) .
$$

Hence $\varrho=\sigma$.

For power series over arbitrary semirings, the sum star identity and the product star identity are valid only for some cycle-free power series.

Theorem 5.2. Let $r, r^{\prime} \in S\left\langle\left\langle\Sigma^{*}\right\rangle\right\rangle$ and assume that $r, r^{*} r^{\prime}$ and $r+r^{\prime}$ are cycle-free. Then the sum star identity is valid for $r$ and $r^{\prime}$.

Proof. We show that $\left(r^{*} r^{\prime}\right)^{*} r^{*}$ is a solution of the cycle-free equation $y=\left(r+r^{\prime}\right) y+\varepsilon$ :

Indeed, by Theorem 3.2 we have $\left(r+r^{\prime}\right)\left(r^{*} r^{\prime}\right)^{*} r^{*}+\varepsilon=r r^{*}\left(r^{\prime} r^{*}\right)^{*}+$ $\left(r^{\prime} r^{*}\right)\left(r^{\prime} r^{*}\right)^{*}+\varepsilon=r r^{*}\left(r^{\prime} r^{*}\right)^{*}+\left(r^{\prime} r^{*}\right)^{*}=r^{*}\left(r^{\prime} r^{*}\right)^{*}=\left(r^{*} r^{\prime}\right)^{*} r^{*}$. We now apply Theorem 5.1.

Theorem 5.3. Let $r, r^{\prime} \in S\left\langle\left\langle\Sigma^{*}\right\rangle\right\rangle$ and assume that $r r^{\prime}$ is cycle-free. Then the product star identity is valid for $r$ and $r^{\prime}$.

Proof. By Theorems 3.2 and 3.1, we obtain $\varepsilon+r\left(r^{\prime} r\right)^{*} r^{\prime}=\varepsilon+r r^{\prime}\left(r r^{\prime}\right)^{*}=$ $\left(r r^{\prime}\right)^{*}$. 
Corollary 5.4. Let $r, r^{\prime} \in S\left\langle\left\langle\Sigma^{*}\right\rangle\right\rangle$ and assume that $r$ is cycle-free and $r^{\prime}$ is proper. Then the sum star identity and the product star identity are valid for $r$ and $r^{\prime}$.

Compare the next lemma with Lemma 4.1.

Lemma 5.5. Let $r, r^{\prime} \in S\left\langle\left\langle\Sigma^{*}\right\rangle\right\rangle$ and assume that $r, r+r^{\prime}$ and $r+r^{\prime} r^{*} r^{\prime}$ are cycle-free. Then

$$
\left(r+r^{\prime}\right)^{*}=\left(r+r^{\prime} r^{*} r^{\prime}\right)^{*}\left(\varepsilon+r^{\prime} r^{*}\right) .
$$

Proof. By our assumptions, the power series $r^{*},\left(r+r^{\prime}\right)^{*}$ and $\left(r+r^{\prime} r^{*} r^{\prime}\right)^{*}$ exist. By Theorem 3.1, we have

$$
\left(r+r^{\prime}\right)^{*}=r\left(r+r^{\prime}\right)^{*}+r^{\prime}\left(r+r^{\prime}\right)^{*}+\varepsilon .
$$

Hence $\left(r+r^{\prime}\right)^{*}$ is a solution of the equation $y=r y+r^{\prime}\left(r+r^{\prime}\right)^{*}+\varepsilon$. By Theorem 5.1 and the cycle-freeness of $r$, another representation of this unique solution is $r^{*} r^{\prime}\left(r+r^{\prime}\right)^{*}+r^{*}$. Substituting $r^{*} r^{\prime}\left(r+r^{\prime}\right)^{*}+r^{*}$ into the third occurrence in the above equality yields

$$
\left(r+r^{\prime}\right)^{*}=\left(r+r^{\prime} r^{*} r^{\prime}\right)\left(r+r^{\prime}\right)^{*}+r^{\prime} r^{*}+\varepsilon
$$

This shows that $\left(r+r^{\prime}\right)^{*}$ is a solution of the equation

$$
y=\left(r+r^{\prime} r^{*} r^{\prime}\right) y+r^{\prime} r^{*}+\varepsilon .
$$

By Theorem 5.1 and the cycle-freeness of $r+r^{\prime} r^{*} r^{\prime}$, another representation for the unique solution of this equation is

$$
\left(r+r^{\prime} r^{*} r^{\prime}\right)^{*}\left(\varepsilon+r^{\prime} r^{*}\right)
$$

Consider matrices $A \in S^{I_{1} \times I_{2}}\left\langle\left\langle\Sigma^{*}\right\rangle\right\rangle$ and $B \in S^{I_{2} \times I_{3}}\left\langle\left\langle\Sigma^{*}\right\rangle\right\rangle$ such that either the matrices $(A, w) \in S^{I_{1} \times I_{2}}$ are row finite for all $w \in \Sigma^{*}$ or the matrices $(B, w) \in S^{I_{2} \times I_{3}}$ are column finite for all $w \in \Sigma^{*}$. Then $A B \in S^{I_{1} \times I_{3}}\left\langle\left\langle\Sigma^{*}\right\rangle\right\rangle$ is well-defined. Hence, for a matrix $A \in S^{I \times I}\left\langle\left\langle\Sigma^{*}\right\rangle\right\rangle$ such that the matrices $(A, w) \in S^{I \times I}$ are row (resp. column) finite for all $w \in \Sigma^{*}$, all powers $A^{n} \in S^{I \times I}\left\langle\left\langle\Sigma^{*}\right\rangle\right\rangle$ are well-defined. If, furthermore, $A$ is cycle-free then $A^{*} \in S^{I \times I}\left\langle\left\langle\Sigma^{*}\right\rangle\right\rangle$ is well-defined.

Lemma 4.1 is the main tool for proving the matrix identities of Theorem 4.2. In an analogous manner, Lemma 5.5 is a main tool for proving-under different assumptions - the same matrix identities in the next theorem.

For the rest of the section, let $I=I_{1} \cup I_{2}$ with $I_{1}, I_{2} \neq \emptyset$ and $I_{1} \cap I_{2}=\emptyset$. The notation is similar to that in Theorem 4.2, but with $A \in S^{I \times I}\left\langle\left\langle\Sigma^{*}\right\rangle\right\rangle$ instead of $A \in S^{I \times I}$. For notational convenience, we will denote in Theorems 5.6, 5.7 and in Corollaries 5.8, 5.9 the matrices $A\left(I_{i}, I_{j}\right)$ by $A_{i, j}$, for $1 \leq i, j \leq 3$. 
Theorem 5.6. Assume that $A \in S^{I \times I}\left\langle\left\langle\Sigma^{*}\right\rangle\right\rangle$ is cycle-free and $(A, w)$ is row and column finite for all $w \in \Sigma^{*}$. Furthermore, assume that $A_{1,1}, A_{2,2}, A_{1,1}+$ $A_{1,2} A_{2,2}^{*} A_{2,1}$ and $A_{2,2}+A_{2,1} A_{1,1}^{*} A_{1,2}$ are cycle-free. Then

$$
\begin{aligned}
& A^{*}\left(I_{1}, I_{1}\right)=\left(A_{1,1}+A_{1,2} A_{2,2}^{*} A_{2,1}\right)^{*}, \\
& A^{*}\left(I_{1}, I_{2}\right)=\left(A_{1,1}+A_{1,2} A_{2,2}^{*} A_{2,1}\right)^{*} A_{1,2} A_{2,2}^{*}, \\
& A^{*}\left(I_{2}, I_{1}\right)=\left(A_{2,2}+A_{2,1} A_{1,1}^{*} A_{1,2}\right)^{*} A_{2,1} A_{1,1}^{*}, \\
& A^{*}\left(I_{2}, I_{2}\right)=\left(A_{2,2}+A_{2,1} A_{1,1}^{*} A_{1,2}\right)^{*} .
\end{aligned}
$$

Proof. Consider the matrices

$$
A_{1}=\left(\begin{array}{cc}
A_{1,1} & 0 \\
0 & A_{2,2}
\end{array}\right) \quad \text { and } \quad A_{2}=\left(\begin{array}{cc}
0 & A_{1,2} \\
A_{2,1} & 0
\end{array}\right) .
$$

Since the blocks of the block-diagonal matrix $A_{1}$ are cycle-free, the matrix $A_{1}^{*}$ exists and equals

$$
A_{1}^{*}=\left(\begin{array}{cc}
A_{1,1}^{*} & 0 \\
0 & A_{2,2}^{*}
\end{array}\right) \text {. }
$$

This implies that

$$
A_{1}+A_{2} A_{1}^{*} A_{2}=\left(\begin{array}{cc}
A_{1,1}+A_{1,2} A_{2,2}^{*} A_{2,1} & 0 \\
0 & A_{2,2}+A_{2,1} A_{1,1}^{*} A_{1,2}
\end{array}\right) .
$$

Since the blocks of the block-diagonal matrix $A_{1}+A_{2} A_{1}^{*} A_{2}$ are cycle-free, the matrix $\left(A_{1}+A_{2} A_{1}^{*} A_{2}\right)^{*}$ exists and equals

$$
\left(A_{1}+A_{2} A_{1}^{*} A_{2}\right)^{*}=\left(\begin{array}{cc}
\left(A_{1,1}+A_{1,2} A_{2,2}^{*} A_{2,1}\right)^{*} & 0 \\
0 & \left(A_{2,2}+A_{2,1} A_{1,1}^{*} A_{1,2}\right)^{*}
\end{array}\right) .
$$

We now apply Lemma 5.5 with $r=A_{1}$ and $r^{\prime}=A_{2}$. The computation of

$$
\left(A_{1}+A_{2} A_{1}^{*} A_{2}\right)^{*}\left(E+A_{2} A_{1}^{*}\right)
$$

proves the theorem.

Theorem 5.7. Consider $A \in S^{I \times I}\left\langle\left\langle\Sigma^{*}\right\rangle\right\rangle$ such that $(A, w)$ is row and column finite for all $w \in \Sigma^{*}$. Furthermore, assume that $A_{1,1}$ and $A_{2,2}$ are cycle-free, and $A_{1,2}$ or $A_{2,1}$ is proper. Then $A$ is cycle-free and

$$
\begin{aligned}
& A^{*}\left(I_{1}, I_{1}\right)=\left(A_{1,1}+A_{1,2} A_{2,2}^{*} A_{2,1}\right)^{*}, \\
& A^{*}\left(I_{1}, I_{2}\right)=\left(A_{1,1}+A_{1,2} A_{2,2}^{*} A_{2,1}\right)^{*} A_{1,2} A_{2,2}^{*}, \\
& A^{*}\left(I_{2}, I_{1}\right)=\left(A_{2,2}+A_{2,1} A_{1,1}^{*} A_{1,2}\right)^{*} A_{2,1} A_{1,1}^{*}, \\
& A^{*}\left(I_{2}, I_{2}\right)=\left(A_{2,2}+A_{2,1} A_{1,1}^{*} A_{1,2}\right)^{*} .
\end{aligned}
$$


Proof. We only prove the case where $A_{2,1}$ is proper. The proof of the other case is similar. An easy proof by induction on $j \geq 1$ shows that

$$
(A, \varepsilon)^{j}=\left(\begin{array}{cc}
\left(A_{1,1}, \varepsilon\right)^{j} & \sum_{j_{1}+j_{2}=j-1}\left(A_{1,1}, \varepsilon\right)^{j_{1}}\left(A_{1,2}, \varepsilon\right)\left(A_{2,2}, \varepsilon\right)^{j_{2}} \\
0 & \left(A_{2,2}, \varepsilon\right)^{j}
\end{array}\right) .
$$

Now let $A_{1,1}$ and $A_{2,2}$ be cycle-free of index $k$. Then $(A, \varepsilon)^{2 k}=0$ and $A$ is cycle-free. Furthermore $\left(A_{1,1}+A_{1,2} A_{2,2}^{*} A_{2,1}, \varepsilon\right)=\left(A_{1,1}, \varepsilon\right)$ and $\left(A_{2,2}+\right.$ $\left.A_{2,1} A_{1,1}^{*} A_{1,2}, \varepsilon\right)=\left(A_{2,2}, \varepsilon\right)$. Hence, the assumptions of Theorem 5.6 are satisfied and our theorem is proved.

Corollary 5.8. Consider $A \in S^{I \times I}\left\langle\left\langle\Sigma^{*}\right\rangle\right\rangle$ such that $(A, w)$ is row and column finite for all $w \in \Sigma^{*}$. Furthermore, assume that $A_{1,1}$ and $A_{2,2}$ are cycle-free and that $A_{2,1}=0$. Then $A$ is cycle-free and

$$
A^{*}=\left(\begin{array}{cc}
A_{1,1}^{*} & A_{1,1}^{*} A_{1,2} A_{2,2}^{*} \\
0 & A_{2,2}^{*}
\end{array}\right) .
$$

Observe that, for finite matrices, the row and column finiteness of $(A, w)$ for all $w \in \Sigma^{*}$ is satisfied and is not needed as assumption in Theorem 5.7. If $A$ is finite and proper, all assumptions of Theorem 5.7 are satisfied.

Corollary 5.9. Let $I$ be finite and $A \in S^{I \times I}\left\langle\left\langle\Sigma^{*}\right\rangle\right\rangle$ be proper. Then

$$
\begin{aligned}
& A^{*}\left(I_{1}, I_{1}\right)=\left(A_{1,1}+A_{1,2} A_{2,2}^{*} A_{2,1}\right)^{*} \\
& A^{*}\left(I_{1}, I_{2}\right)=\left(A_{1,1}+A_{1,2} A_{2,2}^{*} A_{2,1}\right)^{*} A_{1,2} A_{2,2}^{*}, \\
& A^{*}\left(I_{2}, I_{1}\right)=\left(A_{2,2}+A_{2,1} A_{1,1}^{*} A_{1,2}\right)^{*} A_{2,1} A_{1,1}^{*} \\
& A^{*}\left(I_{2}, I_{2}\right)=\left(A_{2,2}+A_{2,1} A_{1,1}^{*} A_{1,2}\right)^{*} .
\end{aligned}
$$

\section{References}

1. J. Adámek, E. Nelson, and J. Reiterman. Tree constructions of free continuous algebras. J. Comput. System Sci., 24:114-146, 1982.

2. J. Berstel, editor. Séries formelles en variables non commutatives et applications. Laboratoire d'Informatique Théorique et Programmation, Ecole Nationale Supérieure de Techniques Avancées, 1978.

3. J. Berstel and C. Reutenauer. Les séries rationelles et leurs langages. Masson, 1984. English translation: Rational Series and Their Languages. EATCS Monographs on Theoret. Comp. Sc., vol. 12, 1988.

4. S. L. Bloom and Z. Ésik. Iteration Theories. EATCS Monographs on Theoret. Comp. Sc. Springer, 1993.

5. G. Carré. Graphs and Networks. Clarendon Press, 1979.

6. J. H. Conway. Regular Algebra and Finite Machines. Chapman \& Hall, 1971.

7. M. Droste and P. Gastin. On aperiodic and star-free formal power series in partially commuting variables. Theory of Computing Systems, 42:608-631, 2008. Extended abstract in: Formal Power Series and Algebraic Combinatorics (D. Krob, A.A. Milchalev, A.V. Milchalev, eds.), 12th Int. Conf., Moscow, Springer, 2000, pp. 158-169. 
8. M. Droste, W. Kuich, and H. Vogler, editors. Handbook on Weighted Automata. Springer, 2009.

9. S. Eilenberg. Automata, Languages and Machines, volume A. Academic Press, 1974.

10. Z. Ésik and W. Kuich. Modern automata theory. www.dmg.tuwien.ac.at/kuich.

11. Z. Ésik and W. Kuich. Locally closed semirings. Monatsh. Math., 137:21-29, 2002.

12. Z. Ésik and W. Kuich. Equational axioms for a theory of automata. In C. Martin-Vide, V. Mitrana, and G. Paun, editors, Formal Languages and Applications, volume 148 of Studies in Fuzziness and Soft Computing, pages 183-196. Springer, 2004.

13. Z. Ésik and W. Kuich. Finite automata. In [8], Chapter 3. 2009.

14. K. Głazek. A Guide to the Literature on Semirings and their Applications in Mathematics and Information Science. Kluwer, 2002.

15. J. A. Goguen, J. W. Thatcher, E. G. Wagner, and J. B. Wright. Initial algebra semantics and continuous algebras. J. Assoc. Comput. Mach., 24:68-95, 1977.

16. J. Golan. Semirings and their Applications. Kluwer Academic Publ., 1999.

17. M. Goldstern. Vervollständigung von halbringen. Diplomarbeit, Technische Universität Wien, 1985.

18. I. Guessarian. Algebraic semantics. In Lect. Notes Comput. Sci., volume 99. Springer, 1981.

19. P. Hájek. Metamathematics of Fuzzy Logic. Kluwer Academic Publ., 1998.

20. U. Hebisch. The Kleene theorem in countably complete semirings. Bayreuther Mathematische Schriften, 31:55-66, 1990.

21. U. Hebisch and H. J. Weinert. Halbringe - Algebraische Theorie und Anwendungen in der Informatik. English translation: Semirings - Algebraic Theory and Applications in Computer Science. World Scientific Publishing, 1998. Teubner, 1993.

22. B. Heidergott, G. J. Olsder and J. van der Woude. Max Plus at Work. Princeton University Press, 2006.

23. G. Karner. On limits in complete semirings. Semigroup Forum, 45:148-165, 1992.

24. G. Karner. Continuous monoids and semirings. Theoretical Computer Science, 318:355-372, 2004

25. D. Kozen. A completeness theorem for Kleene algebras and the algebra of regular events. Inf. Computation, 110:366-390, 1994.

26. D. Krob. Monoides et semi-anneaux complets. Semigroup Forum, 36:323-339, 1987.

27. D. Krob. Monoides et semi-anneaux continus. Semigroup Forum, 37:59-78, 1988.

28. W. Kuich. The Kleene and the Parikh theorem in complete semirings. In ICALP'87, volume 267 of Lect. Notes Comput. Sci., pages 212-225, 1987.

29. W. Kuich. Semirings and formal power series: Their relevance to formal languages and automata theory. In G. Rozenberg and A. Salomaa, editors, Handbook of Formal Languages, volume 1, chapter 9, pages 609-677. Springer, 1997.

30. W. Kuich and A. Salomaa. Semirings, Automata, Languages, volume 5 of EATCS Monographs on Theoretical Computer Science. Springer, 1986.

31. D. J. Lehmann. Algebraic structures for transitive closure. Theoretical Computer Science, 4:59-76, 1977. 
32. E. G. Manes and M. A. Arbib. Algebraic Approaches to Program Semantics. Springer, 1986

33. G. Markowsky. Chain-complete posets and directed sets with applications. Algebra Universalis, 6:53-68, 1976.

34. M. Mohri. Semiring frameworks and algorithms for shortest-distance problems. J. Automata, Languages and Combinatorics, 7:321-350, 2002.

35. I. Petre and A. Salomaa. Algebraic systems and pushdown automata. In [8], Chapter 7. 2009.

36. G. Rahonis. Fuzzy languages. In [8], Chapter 12. 2009.

37. J. Sakarovitch. Kleene's theorem revisited. In Selected contributions on Trends, techniques, and problems in theoretical computer science, 4th International Meeting of Young Computer Scientists, volume 281 of Lect. Notes Comput. Sci., pages 39-50, 1987.

38. J. Sakarovitch. Éléments de Théorie des Automates. Vuibert, 2003.

39. J. Sakarovitch. Rational and recognisable power series. In [8], Chapter 4. 2009.

40. A. Salomaa and M. Soittola. Automata-Theoretic Aspects of Formal Power Series. Springer, 1978.

41. W. Wechler. The Concept of Fuzziness in Automata and Language Theory. Akademie-Verlag, 1978.

42. X. Zhao. Locally closed semirings and iteration semirings. Monatsh. Math., 144:157-167, 2005.

43. U. Zimmermann. Linear and Combinatorial Optimization in Ordered Algebraic Structures, volume 10 of Annals of Discrete Mathematics. North Holland, 1981. 